\title{
Nonlinear screening in large two-dimensional Coulomb clusters
}

\author{
Minghui Kong, A. Vagov, B. Partoens, and F. M. Peeters* \\ Departement Fysica, Universiteit Antwerpen (Campus Drie Eiken), Universiteitsplein 1, B-2610 Antwerpen, Belgium \\ W. P. Ferreira and G. A. Farias \\ Departamento de Física, Universidade Federal do Ceará, Caixa Postal 6030, Campus do Pici, 60455-760 Fortaleza, Ceará, Brazil
}

(Received 22 June 2004; published 22 November 2004)

\begin{abstract}
The distortion due to a fixed point impurity with variable charge placed in the center of a classical harmonically confined two-dimensional (2D) large Coulomb cluster is studied. We find that the net topological charge $\left(N_{-}-N_{+}\right)$of the system is always equal to six independent of the position and charge of the impurity. In comparison with a 2D cluster without impurity charge, only the breathing mode remains unchanged. The screening length is found to be a highly nonlinear function of the impurity charge. For values of the impurity charge smaller than the charge of the other particles, the system has almost the same screening strength. When the impurity charge is larger, the screening length is strongly enhanced. This result can be explained by the competition between the different forces active in the system.
\end{abstract}

DOI: 10.1103/PhysRevE.70.051807 PACS number(s): 61.46. +w, 73.22.-f, 36.40.Sx, 52.27.Lw

\section{INTRODUCTION}

Wigner-like ordered systems have been the subject of intense interest over the past few decades. Systems consisting of a finite number of particles with repulsive interparticle interaction which are kept together by an external confinement potential have been realized recently. Typical experimental realizations of such 2D systems include electrons on the surface of liquid helium [1], electrons in quantum dots [2], colloidal suspensions [3] and confined plasma crystals [4].

Colloids and dust particles are sufficiently large that the particle movement can directly be observed by an optical microscope. These systems are extensively studied to understand the freezing and melting; and the fundamental dynamic processes, i.e., phonon propagation and normal modes. An interesting feature in such finite size systems is the appearance of topological defects. Unlike ordinary solids which have complicated interparticle potentials, in such systems the interparticle interactions are in most cases precisely known and easily controlled by the experiment. Much experimental [5-7] and theoretical work [8-13] was devoted to the study of those topological defects in an infinite 2D classical Wigner crystal and recently also in a finite size system.

Classical point charges in a 2D infinite plane crystallize into a hexagonal lattice at low temperatures [14]. When the particles are confined by an external parabolic potential, there is a competition between the interparticle potential to form the bulk hexagonal lattice and the ringlike structure at the edge imposed by the circular confinement potential [15]. In the transition region defects appear as dislocations and disclinations at the six corners of the hexagonal-shaped inner domain in the specific case of pure Coulomb interaction [13].

However, in real experiments clusters are not always clean, impurities and/or defect particles with deviating mass

*Electronic address: francois.peeters@ua.ac.be and/or charge can be present. It will be very interesting to know the general role of the impurity potential on the ordered (lattice) structure. How such an impurity potential affects essential characteristics of a system such as, the topological charge, the density of states (DOS) of the normal modes, and the normal modes of the system, requires extensive studies.

Recently, the influence of a single mobile defect particle on the structure of a small classical 2D cluster was investigated [16], which explained recent experimental results of the configurations of a system of rotating magnetic disks [6]. Theoretical work on the structure of classical clusters consisting of two-species of charged particles with the same mass was reported recently [17], where it was found that the species with the largest charge move towards the outer edge of the system. Recently, the analog quantum system (also called quantum dot) was studied in Refs. $[18,19]$ in the case of a small number of electrons. Interesting, reentering of the Wigner crystal state was predicted [18] as a function of the magnetic field. Very recently, nonlinear quantum screening in $2 \mathrm{D}$ electron gases was also investigated in Ref. [20] by using the density functional theory method.

In this work, we study the screening of a single fixed impurity (defect) with variable charge in a large 2D classical Coulomb system which is confined by a circular parabolic potential. This case is essentially different from the previous studied system [16]: (1) The impurity is immobile, i.e., has an infinite mass, and (2) we consider large systems. Also unlike the study of Ref. [17] this impurity can have an arbitrary charge. In contract to Refs. $[16,17]$ we will also investigate the topological charge and the normal modes of the system.

The present paper is organized as follows. In Sec. II, the model system and the numerical approach are described. Section III is devoted to the ground state configuration and topological defects. The normal modes are presented in Sec. IV. In Sec. V, the nonlinear screening of the impurity charge in these clusters are discussed. Our conclusions are given in Sec. VI. 


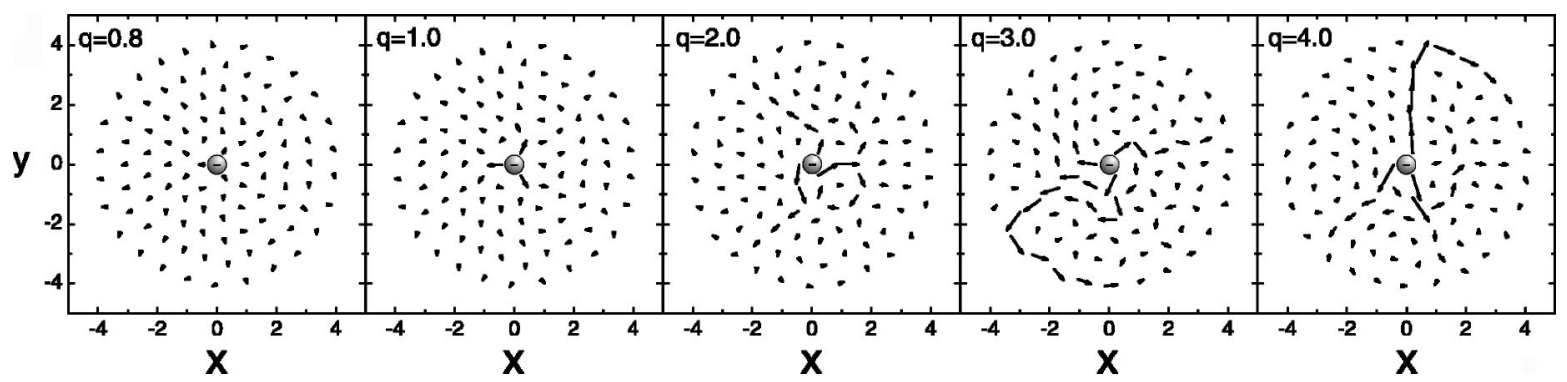

FIG. 1. The displacement of the particles for different values of the impurity charge $q$. The impurity is situated in the center and its position is indicated by the solid dot. The arrows show the direction and length of the particles' displacement.

\section{NUMERICAL APPROACH}

The Hamiltonian of a 2D system of $N$ equally charged particles in the presence of a single impurity with arbitrary charge placed in a parabolic confinement potential and interacting through pure Coulomb interaction is given by

$$
H=\sum_{i=1}^{N} \frac{1}{2} m \omega_{0}^{2} r_{i}^{2}+\frac{e^{2}}{\varepsilon} \sum_{i>j}^{N} \frac{1}{\left|\vec{r}_{i}-\vec{r}_{j}\right|}+\frac{e^{2}}{\varepsilon} \sum_{i=1}^{N} \frac{q}{\left|\vec{r}_{i}-\vec{r}_{q}\right|},
$$

where $m$ is the mass of the mobile particles, $\omega_{0}$ the radial confinement frequency, $\varepsilon$ the dielectric constant of the medium the particles are moving in, $e$ the particle charge, $q$ the impurity charge located at $\vec{r}_{q}$, and $\vec{r}_{i}=\left(x_{i}, y_{i}\right)$ the position of the $i$ particle with $r_{i} \equiv\left|\vec{r}_{i}\right|$, where the third term in Eq. (1) corresponds to the interaction between the mobile identical particles and the single fixed impurity with charge $q$.

We can write the Hamiltonian in dimensionless form if we express the coordinates and energy in the following units [15]: $r_{0}=\left(e^{2} / \varepsilon / \alpha\right)^{1 / 3}, E_{0}=\left(e^{2} / \varepsilon\right)^{2 / 3} \alpha^{1 / 3}$, with $\alpha=m \omega_{0}^{2} / 2$. The dimensionless Hamiltonian is given by

$$
H=\sum_{i=1}^{N} r_{i}^{2}+\sum_{i>j}^{N} \frac{1}{\left|\vec{r}_{i}-\vec{r}_{j}\right|}+\sum_{i=1}^{N} \frac{q}{\left|\vec{r}_{i}-\vec{r}_{q}\right|} .
$$

To find the thermodynamic equilibrium configurations, we employed the Newton optimization technique after the standard Monte Carlo (MC) routine [21]. This procedure was outlined and compared with the standard MC technique in Ref. [22]. The eigenmode frequencies are obtained from the square root of the eigenvalues of the dynamical matrix [22]

$$
H_{\alpha \beta, i j}=\left.\frac{\partial^{2} H}{\partial r_{\alpha, i} \partial r_{\beta, j}}\right|_{r_{\alpha, i}=r_{\alpha, i}^{n}},
$$

where $\left\{r_{\alpha, i}^{n}, \alpha=x, y ; i=1, \ldots, N\right\}$ is the position of the particles in the ground state configuration. The eigenfrequencies in this paper will be expressed in units $\omega^{\prime}=\sqrt{2} \omega_{0}$.

\section{GROUND STATE CONFIGURATIONS AND TOPOLOGICAL DEFECTS}

In order to investigate the influence of the impurity, we consider clusters which are large enough to show a hexagonal lattice structure in the center. This is realized starting from around $N=100$ particles, although larger systems will also be simulated.
First, the ground state of a cluster with equal charges with one impurity fixed in the center is obtained. The displacement of the particles due to the presence of the impurity which is located in the center of the cluster is shown in Fig. 1 for $N=100$ particles and different values of the charge $q$ of the impurity. The vectors show the direction and length of the displacement of the particles as a consequence of the introduction of the impurity. From Fig. 1, we notice that when the impurity charge $q \leqslant 1$, only the center particles which are very close to the impurity are mainly displaced, in spite of the long range Coulomb interaction between the particles. In fact, a very small screening length was observed in this case. This will be discussed in more details in the next section. For larger values of the impurity charge, more particles are influenced. As can be seen in Fig. 1, the particles undergo vortex-like movements. This is a consequence of the circular confinement potential which keeps the particles in a finite region. Such a motion does not lead to any compression and guarantees the same density. For large values of the impurity charge the distortion in the lattice is larger and it propagates further away from the center of the cluster.

Next we resort to the Voronoi construction [23] in order to investigate the ordering of the particles and to reveal the topological nature of the particles arrangement. A detailed study of the topological defects in a confined 2D Coulomb cluster, in the absence of the impurity, was previously reported in Ref. [13]. These defects appear as a consequence of the adjustment of a triangular lattice to a circular region. However, when an impurity charge is present, and depending on its value, the number of defects increases.

In Fig. 2 we show the Voronoi constructions of the ground-state configuration of a cluster with $N=100$ particles and with an impurity charge of variable value fixed at the point $(0,0)$. The quantity $N_{-}$(indicated by "-") means an orientational defect with fivefold coordination number, while the quantity $N_{+}$(indicated by "+") means an orientational defect with seven fold coordination number (the number of sides of the polygon around the particles is nothing else then the coordination number). In the absence of the impurity charge, the ground state configuration of the system with $N$ $=100$ particles presents the minimum number of defects, i.e., six, which is equal to the net topological charge. In addition, all topological defects are located at the transition region which is between the central hexagonal structure and the outer rings.

When the system has one identical impurity charge ( $q$ $=1$ ) at the center, the number of defects is still equal to six, 


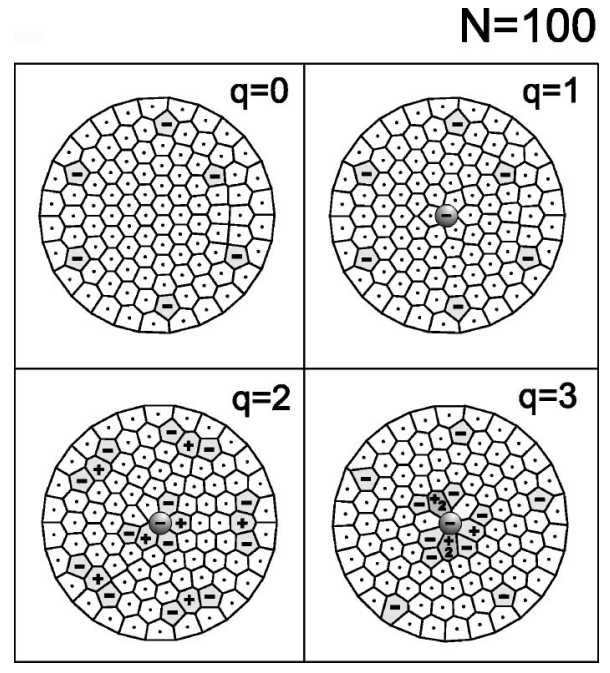

FIG. 2. The Voronoi structure where the defects (i.e., disclinations) are indicated by " $2+$ " for an 8 -fold, "+" for a 7-fold and by "-" for a 5-fold coordination number.

and all of them are located in the transition region of the cluster. For larger values of the impurity charge, topological defects start to appear in the center region of the cluster, and the lattice distortion gets bigger. Because a large impurity charge will strongly repel the neighboring particles, an empty space will appear in the center region. As a consequence the hexagonal lattice structure is strongly distorted and more defects will appear around this circle. Notice that some defects near the circle can have an eightfold coordinate number (indicated by “ +2 "), which does not happen in $2 \mathrm{D}$ clusters in the absence of the impurity charge. However, we want to stress that for all values of the impurity charge, the net topological charge $N_{-}-N_{+}$is always equal to six.

Another interesting phenomena is that the total number of defects $\left(N_{-}+N_{+}\right)$first increases with increasing impurity charge till $q \simeq 2.4$. Further increasing the impurity charge leads to a decrease of the total number of defects. This can be explained together with the nonlinear screening behavior in the next section by the competition between the different forces active in the system.

In Fig. 3 we present the Voronoi construction for the ground state configurations in the cases of two particular values of the impurity charge, i.e., $q=1$ and $q=2$. In addition

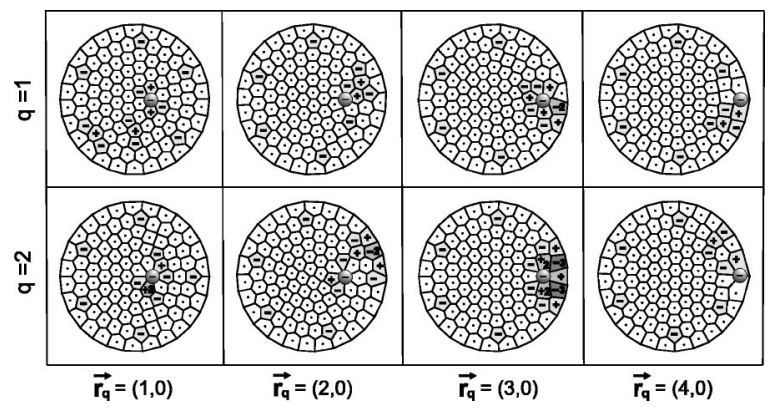

FIG. 3. The same as Fig. 2 but where now the impurity charge is displaced from its central location. The upper (lower) row of figures corresponds to an impurity with single (double) charge and results are given for different positions of the impurity $\vec{r}_{q}$.

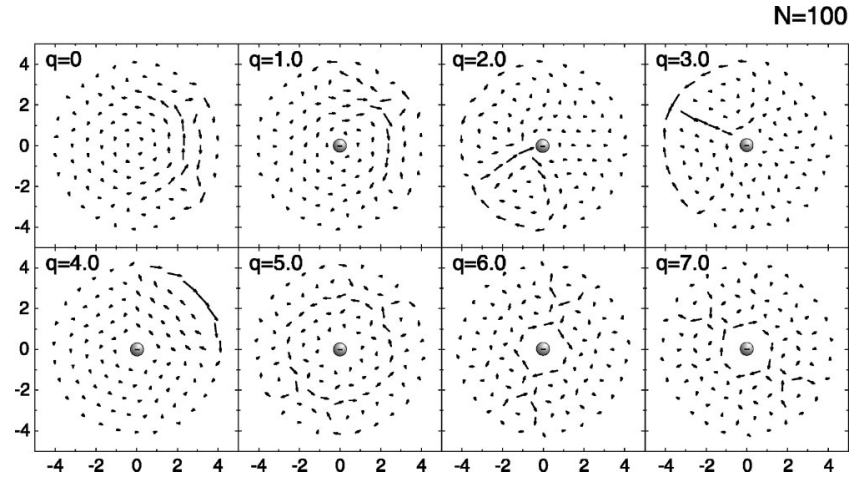

FIG. 4. Vector plot of the lowest nonzero eigenfrequency for the cluster with $N=100$ particles for different values of the impurity charge $q$.

the impurity's position is moved away from the center of the system. As can be observed, the net topological charge $N_{-}$ $-N_{+}$of the system, in spite of the different values and positions of the impurity charge is always equal to six.

These results are in agreement with the Euler theorem [8], which is based on the circular symmetry of the confinement potential. It is clear that the presence of the impurity does not change the net topological charge.

\section{NORMAL MODES}

For systems without impurity, it is known that the lowest nonzero excitation corresponds with an intershell rotation for small clusters and with a vortex-antivotex motion for large clusters [22]. Figure 4 shows some examples of these lowest nonzero frequency (LNF) modes for $N=100$ particles in the presence of an impurity charge $q$. Notice that for $q<5$, a vortex-antivortex excitation still corresponds with the LNF, while for larger values of the impurity charge $(q \geqslant 5)$ the LNF mode corresponds to a circular motion around the impurity and concomitant smaller local circular motions near the edge.

For a parabolic confinement, it is well known that there are three eigenfrequencies which are independent of $N$ [22]: $\omega=0, \sqrt{2}$ and $\sqrt{6}$, which correspond to the rotation of the system as a whole, the center of mass (c.m.) mode and the breathing mode (BM), respectively. The BM mode was recently measured experimentally $[24,25]$. In the system with a central impurity, the rotation of the system as a whole does not change, because the symmetry of the system is not broken. The c.m. mode is destroyed by this fixed impurity and the new mode will go around the impurity in the center, so it can no longer be called a c.m. mode.

However, the BM mode with $\omega / \omega^{\prime}=\sqrt{6}$ still survives and its value does not depend on the charge of the impurity. This can also be obtained analytically. From Eq. (2), the Hamilton equation of motion yields

$$
\dot{v}_{x i}=-\frac{\partial H}{\partial x_{i}}=-2 x_{i}+\frac{x_{i}-x_{j}}{\left|\mathbf{r}_{i}-\mathbf{r}_{j}\right|^{3}}+q \sum_{i \neq 1}^{N} \frac{x_{i}-x_{q}}{\left|\mathbf{r}_{i}-\mathbf{r}_{q}\right|^{3}} .
$$

Here the impurity is fixed in the center, so $\vec{r}_{q} \equiv\left(x_{q}, y_{q}\right)$ $=(0,0)$. With this result, one obtained for the mean square radius $R^{2}=\Sigma_{1}^{N}\left(x_{i}^{2}+y_{i}^{2}\right)$, then 

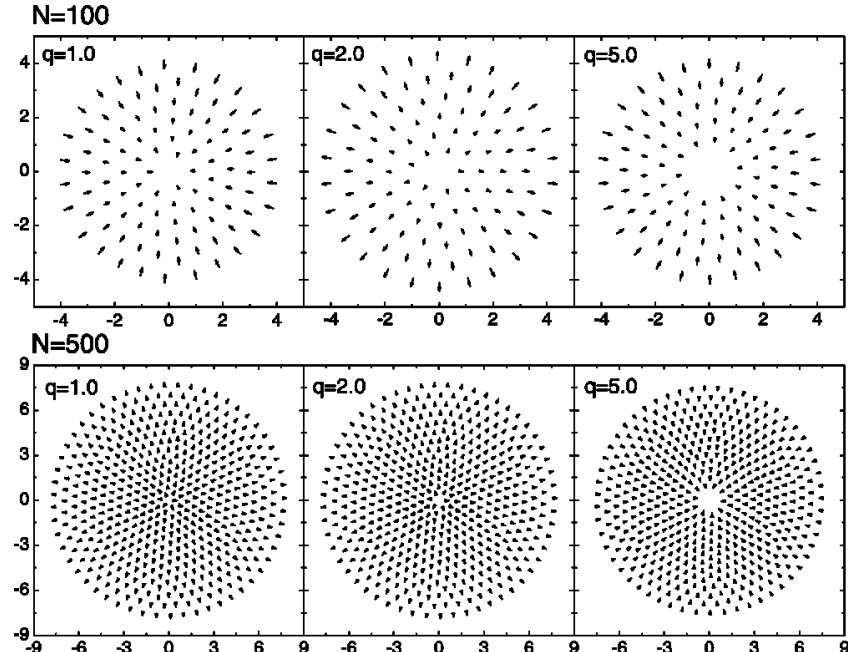

FIG. 5. The breathing modes for some particular values of the impurity charge $q$ for the clusters with $N=100$ and $N=500$ particles. In all cases, the BM has the same frequency $\omega^{\prime}=\sqrt{6}$.

$$
\frac{d^{2} R^{2}}{d t^{2}}=-6 R^{2}+\frac{2}{N}(H+T),
$$

with $T=\sum_{i}\left(\dot{x}_{i}^{2}+\dot{y}_{i}^{2}\right)$ the total kinetic energy. So we see that the breathing mode still survives with frequency $\sqrt{6}$ for the system with a fixed central impurity.

We plot in Fig. 5 several typical cases for the breathing mode for $N=100$ and $N=500$ particles in the case the impurity is located in the center of the confinement potential. But when the impurity is not in the center, the BM is strongly modified and its frequency is different from $\sqrt{6}$.

The density of states (DOS) of the normal modes (phonons), in the absence of the impurity, was investigated before [22]. This is obtained by a summation of the energy levels within some small energy region. It shows that all large clusters have two clear broad maxima which is in qualitative agreement with the case for an infinite system [14]. We compare the DOS for a large system with $N=500$ particles with the situation for a fixed central impurity with two different values of its charge, i.e., $q=1,2.6$. Here a larger system with $N=500$ particles is considered and we take $\delta \omega$ $=\omega_{\max } / 20$ as the frequency interval, where $\omega_{\max }$ is the maximum eigenfrequency. In Fig. 6, one can see that the system's DOS does not change too much in these two cases with or without the fixed impurity. As we know, a finite confined cluster has compressionlike and shearlike modes. The compressional and shearlike properties can be extracted from the divergence and rotor of the velocity field, respectively. In this paper, we will associate a single number to the shearlike and compressionlike character of the different modes by calculating the spatial average of the square of the divergence $\vec{\nabla} \cdot \vec{v}$ and the rotor $(\vec{\nabla} \times \vec{v})_{z}$ of the velocity field, following the approach of Refs. [22,26,27].

We plotted in Fig. 7 the divergence $\psi_{d}(k)$ and rotor $\psi_{r}(k)$ as a function of the excitation frequency for $N=500$ particles for different values of the charge of the impurity. One can see from this picture that the system does not change quali-

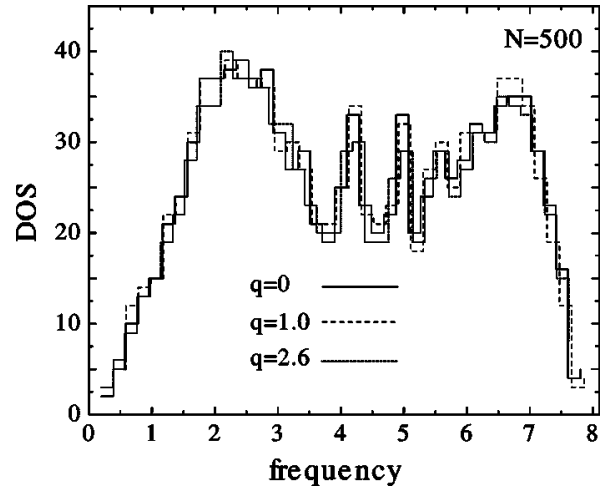

FIG. 6. Density of phonon states for clusters with $N=500$ particles in the presence of a central impurity for different values of the impurity charge $q$.

tatively its overall shearlike and compressionlike character when an impurity is present even when the impurity charge is increased.

\section{NONLINEAR SCREENING}

Screening of the impurity charge is a fundamental property of a gas of charge carriers. Such a screening is characterized by the displacement of the carriers when an impurity is placed in a uniform gas of carriers [28].

We investigate the behavior of the screening length as function of the charge of the impurity for a large 2D cluster with $N=500$ charged particles. In order to quantify the influence of the impurity charge on the system, we will consider here an average deviation of the particles' position before

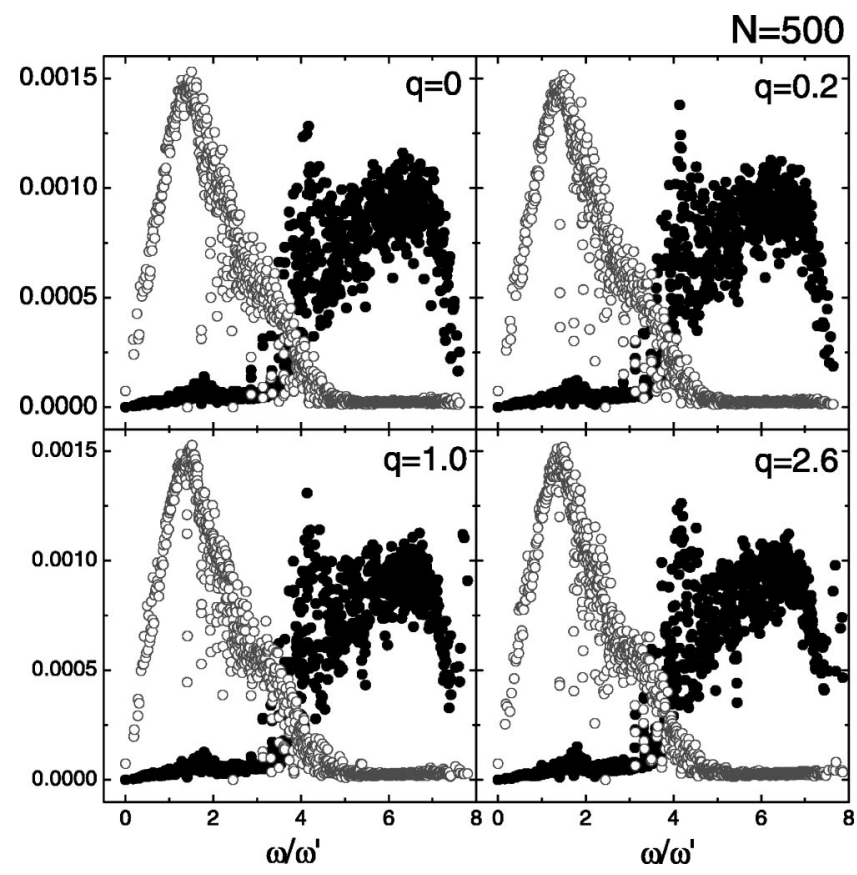

FIG. 7. Divergence $\psi_{d}(k)$ (solid dots) and rotor $\psi_{r}(k)$ (open dots) as a function of the excitation frequency for $N=500$ particles for different values of the impurity charge $q$. 


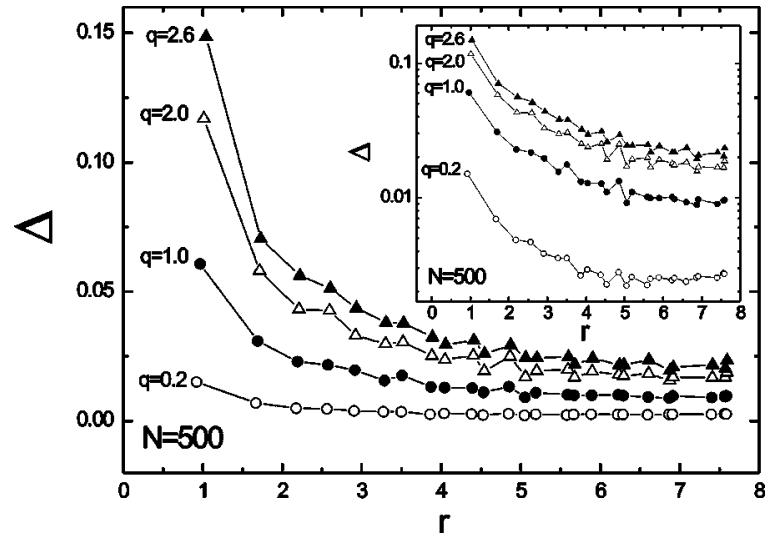

FIG. 8. The deviation $\Delta$ of the radial position of the particles as function of $r$ for different values of the impurity charge $q$ for a system consisting of $N=500$ particles. The inset shows $\Delta$ in a logarithmic scale.

and after the impurity charge is placed in the system. To do so, we initially define, for each particle, the displacement due to the presence of the impurity charge $\Delta_{i}=\left|\overrightarrow{\mathbf{r}}_{i}^{\text {after }}-\overrightarrow{\mathbf{r}}_{i}^{\text {before }}\right|$.

The system is divided into 25 concentric rings with a certain width each containing 20 particles. In every ring $i$ an average displacement is calculated $\Delta_{j}=\Sigma \Delta_{i} / 20$ where the summation $i$ sums over the particles in ring $j$. In this way we obtain the average deviation $\Delta$ as a function of the distance in the radial direction from the center of the cluster.

In Fig. 8, we present $\Delta$ as a function of the distance along the radial direction $r$, for different values of the impurity charge $q$ in a cluster with $N=500$ particles. The inset shows the same plot, but in a logarithmic scale. As can be observed, the edge particles are much less affected by the impurity charge than the central particles. The interaction between the impurity and the particles clearly presents two different regimes, which are related to the value of the impurity charge. When $q<1$, the quantity $\Delta$ becomes almost zero at the extremity of the cluster, indicating that the interaction is strongly screened. On the other hand, when $q>1$, the quantity $\Delta$ is considerable larger even at the edge of the cluster. Notice also that the value of $\Delta$ at the border of the system seems to saturate when $q>2$.

We fit these deviation curves $\Delta(r)$ using the function (i.e., Yukawa potential formation)

$$
\Delta r_{i}=\alpha\left(e^{-r_{i} / \lambda} / r_{i}\right)+r_{0},
$$

where $\lambda$ is the screening length, $\alpha$ a constant and $r_{0}$ is a measure of the expansion of the system as a whole. The results for the screening length as a function of the impurity charge $q$ are shown in Fig. 9. There is a clear nonlinear relationship between the screening length $\lambda$ and the impurity charge $q$. The screening length is almost constant for $q<0.5$, it rapidly increases with increasing value of the impurity charge, in the interval $0.5 \leqslant q \leqslant 1$. For $1.0 \leqslant q \leqslant 1.2$, the screening length reaches its maximum value. When $q>1.2$, the screening parameter decreases slowly with increasing value of the impurity charge. In the inset of Fig. 9, we notice that the $r_{0}-q$ dependence exhibits three different regimes

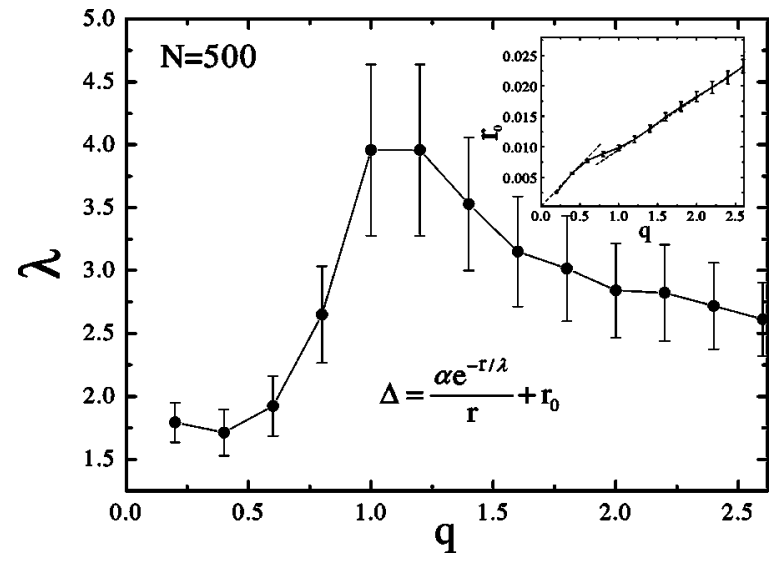

FIG. 9. The screening length as a function of the impurity charge $q$ for clusters consisting of $N=500$ particles. The inset shows the $q$ dependence of the radial displacement at large distances from the impurity. The dotted lines show the extrapolated small and large $q$ behavior.

with a $r_{0}-q$ linear dependence for $q<0.5$ and $q>1$, and a transition region for $0.5<q<1$.

This nonlinear screening behavior can be understood as follows. From Fig. 1, we know that for small values of $q$ till $q<1.2$ the particles near the central region have much larger displacements than the particles near the edge, which are less influenced by the external impurity charge. In this case the system is strongly screened. By increasing the impurity charge $q$, the radius of the system grows linearly [see Fig. 10(b)]. However, the displacement of the particles is not homogeneous in the clusters. The maximum of the screening
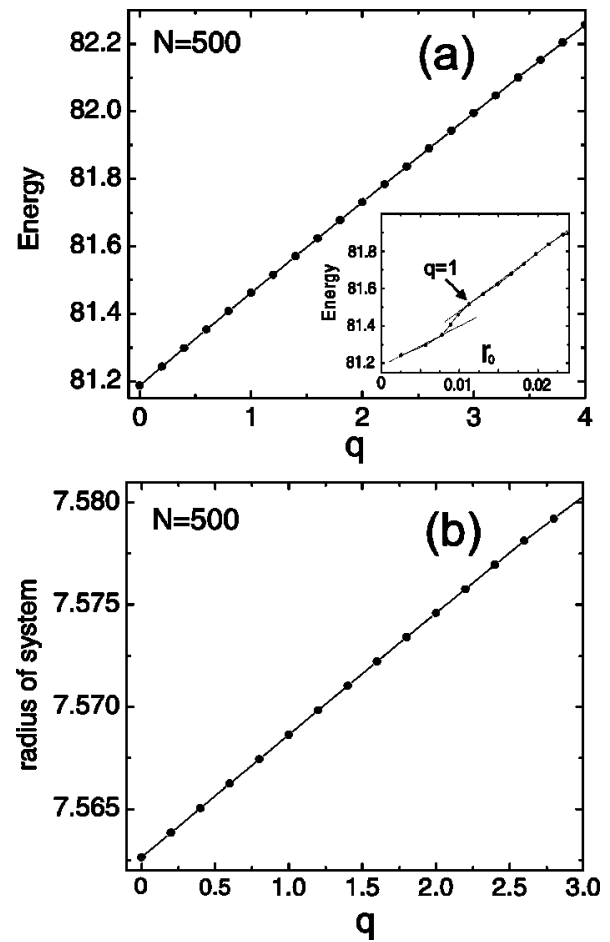

FIG. 10. The energy (a) and radius (b) of the system as a function of the impurity charge $q$. The inset of (a) shows the energy of the system as a function of $r_{0}$. 
length is reached for $q=1.0-1.2$. In this case the difference between the displacements of particles around the center and the edge is largest. At the same time, more defects will appear in the system. Further increasing the impurity charge $q$ leads more and more to a homogenous displacement in all regions (i.e., a central hole is created by the impurity as is clearly seen in Fig. 4 and 5), leading to a decrease of the screening length.

The nonlinear behavior of the screening length is a consequence of the balance among the effect of the impurity repulsive force, the interaction between the charged particles of the system, and the circular confinement potential.

We plot the energy and outer radius of the system as a function of the impurity charge $q$ in Fig. 10. In the inset of Fig. 10(a), the energy of the system as a function of $r_{0}$ is shown. Notice that both the energy and the radius of the system present an almost linear dependence on the impurity charge. The size of the system linearly increases with the impurity charge [Fig.10(b)]. Notice that the outer radius and the uniform increment $r_{0}$ [see Eq. (6)] do not have a one-toone relation because of the first term on the right hand side of Eq. (6) which is different from zero at the edge of the system. From the inset of Fig. 10(a), we notice two regimes with a different linear $E-r_{0}$ dependence. The transition region occurs for $r_{0} \simeq 0.01$ which corresponds to $q \simeq 1$ and this is just the region where $\lambda$ is maximal.

\section{CONCLUSIONS}

We investigated 2D large clusters consisting of identical charged classical particles having a single probe impurity.
All interactions are pure Coulombic. The ground-state configuration is obtained through the Monte Carlo simulation technique. The presence of an impurity charge in the system does not modify the net topological charge $\left(N_{-}-N_{+}\right)$of the system, which is always equal to six. In comparison with a 2D cluster without impurity charge, only the breathing mode remains unchanged if the impurity is located in the center of the confinement potential. The DOS and the shearlike or compressionlike character of normal modes of the system do not change considerably by this impurity as well. A clear nonlinear relationship between the screening length $\lambda$ and the impurity charge is found. For values of the impurity charge smaller than the charge of the other particles, the system has almost a screening strength independent on the charge of the impurity. For larger values of the impurity charge, the system exhibits a clear nonlinear screening length. This result can be explained by the competition between the different forces active in the system.

\section{ACKNOWLEDGMENTS}

This work was supported by the Flemish Science Foundation (FWO-Vl), the Belgian Science Policy, the "Onderzoeksraad van de Universiteit Antwerpen" (GOA), and the EU Research Training Network on "Surface Electrons on Mesoscopic Structures." B.P. and A.V. were supported by the FWO-Vl. G.A.F. was supported by the Brazilian National Research Council (CNPq). The authors are very grateful to Dr. L. R. E. Cabral for helpful discussions.
[1] C. C. Grimes and G. Adams, Phys. Rev. Lett. 42, 795 (1979).

[2] R. C. Ashoori, Nature (London) 379, 413 (1996).

[3] M. Golosovsky, Y. Saado, and D. Davidov, Phys. Rev. E 65, 061405 (2002).

[4] J. H. Chu and L. I, Phys. Rev. Lett. 72, 4009 (1994).

[5] C. H. Chiang and L. I, Phys. Rev. Lett. 77, 647 (1996).

[6] B. A. Grzybowski, X. Jiang, H. A. Stone, and G. M. Whitesides, Phys. Rev. E 64, 011603 (2001).

[7] W. Y. Woon and L. I, Phys. Rev. Lett. 92, 065003 (2004).

[8] A. A. Koulakov and B. I. Shklovskii, Phys. Rev. B 57, 2352 (1998).

[9] Y. J. Lai and L. I, Phys. Rev. E 60, 4743 (1999).

[10] I. V. Schweigert, V. A. Schweigert, and F. M. Peeters, Phys. Rev. Lett. 82, 5293 (1999).

[11] Y. J. Lai and L. I, Phys. Rev. E 64, 015601 (2001).

[12] C. Reichhardt and C. J. O. Reichhardt, Phys. Rev. Lett. 90, 095504 (2003).

[13] M. Kong, B. Partoens, and F. M. Peeters, Phys. Rev. E 67, 021608 (2003).

[14] L. Bonsall and A. A. Maradudin, Phys. Rev. B 15, 1959 (1977).

[15] V. M. Bedanov and F. M. Peeters, Phys. Rev. B 49, 2667 (1994).
[16] K. Nelissen, B. Partoens, and F. M. Peeters, Phys. Rev. E 69, 046605 (2004).

[17] J. A. Drocco, C. J. Olson Reichhardt, C. Reichhardt, and B. Jankó , Phys. Rev. E 68, 060401 (2003).

[18] B. Szafran and F. M. Peeters, Europhys. Lett. 66, 701 (2004).

[19] B. Reusch and R. Egger, Europhys. Lett. 64, 84 (2003).

[20] E. Zaremba, I. Nagy, and P. M. Echenique, Phys. Rev. Lett. 90, 046801 (2003).

[21] N. Metropolis, A. W. Rosenbluth, M. N. Rosenbluth, A. M. Teller, and E. Teller, J. Chem. Phys. 21, 1087 (1953).

[22] V. A. Schweigert and F. M. Peeters, Phys. Rev. B 51, 7700 (1995).

[23] S. Fortune, Algorithmica 2, 153 (1987).

[24] A. Melzer, M. Klindworth, and A. Piel, Phys. Rev. Lett. 87, 115002 (2001).

[25] T. E. Sheridan, C. R. Buckey, D. J. Cox, R. J. Merrill, and W. L. Theisen, Phys. Lett. A 329, 88 (2004).

[26] A. Melzer, Phys. Rev. E 67, 016411 (2003).

[27] M. Kong, B. Partoens, and F. M. Peeters, New J. Phys. 5, 23 (2003).

[28] T. Ando, A. B. Fowler, and F. Stern, Rev. Mod. Phys. 54, 437 (1982). 